\title{
La cara oculta de los advergames. La explotación crítica de un nuevo recurso de la publicidad
}

\author{
Cristina Martorell | cristinamc4@blanquerna.url.edu \\ Josep A. Rom I joseprr@blanquerna.url.edu \\ UNIVERSITAT RAMON LLULL
}

Resumen: En los últimos años, los advergames -videojuegos creados para promocionar productos o marcas- han demostrado ser una forma lúdica, amena y no intrusiva de transmitir mensajes publicitarios a los consumidores. Sin embargo, diversas organizaciones y movimientos contraculturales también han empezado a utilizar estos formatos con fines opuestos, es decir, para vehicular sus críticas hacia determinadas marcas, compañías, ideologías o actitudes. En este artículo se pretende profundizar sobre este tipo de videojuegos que actúan como un altavoz anti-publicitario y/o contracultural, los llamados anti-advergames, a través de dos casos prácticos -la crítica a McDonald's a partir del anti-advergame McVideoGame, y la reflexión sobre la política exterior del ex-presidente Bush mediante el videojuego September 12-.

Palabras clave: advergames, anti-advergames, nuevas formas publicitarias

Abstract: In recent years, advergames -videogames created to promote products or brands- have proved to be an entertaining, funny and non-interruptive way to reach consumers. However, some countercultural and anti-consumerism movements have also begun to use these tools at cross purposes, that is to say, in order to criticize certain attitudes from brands and companies. This article aims to deepen on this kind of videogames against advertising and/or mainstream culture, known as antiadvergames, through two case studies -the criticism to McDonald's business policies through the antiadvergame McVideoGame, and the reflection about ex-president Bush "war on terror" consequences through the anti-advergame September 12-.

Key words: advergames, anti-advergames, new advertising techniques 


\section{Introducción}

No existe réplica sin contrarréplica. La comunicación persuasiva no ceja en su eterno empeño de abrir los flancos de la publicidad a nuevas formas de crear discursos atractivos y eficaces para mejorar la conexión con los públicos. Pero a la cultura le corresponde la contracultura y a la publicidad la anti-publicidad. La dialéctica de la sociedad se construye mediante un juego de espejos.

En un entorno mediático como el actual, las empresas buscan sin cesar nuevas fórmulas para conectar con sus consumidores potenciales, entre las que se halla el uso de estrategias de comunicación pull. Estas estrategias, en vez de interrumpir a su público objetivo, optan por atraerlo ofreciéndole contenidos de su interés -en forma de información, entretenimiento o diversión, como es el caso de los advergames.

Esta herramienta de comunicación, resultado de la convergencia entre publicidad y videojuegos, aún se encuentra en una fase de desarrollo y aplicación muy embrionaria. Sin embargo, esto no ha impedido que desarrolladores de videojuegos y activistas hayan definido ya las bases de su contrarréplica, los denominados anti-advergames.

En este artículo se pretende profundizar sobre el fenómeno de los anti-advergames, que usan los videojuegos como un altavoz anti-publicitario y/o contracultural, a través de dos casos prácticos -la crítica a McDonald's a partir del anti-advergame McVideoGame, y la crítica a la política exterior del ex-presidente Bush mediante el videojuego September 12-.

\section{El nuevo paradigma publicitario: de la interrupción a la invitación}

Progresivamente, y debido a circunstancias diversas -tales como la generalización de la banda ancha, la madurez de los consumidores o la democratización de los contenidos-, el sector publicitario empieza a replantearse la efectividad de interrumpir a sus consumidores potenciales mediante estrategias de comunicación push -publicidad no solicitada inserta en los medios de comunicación tradicionales-.

Por ello, cada vez más se opta por "invitarlos" a que se interesen por lo que puede ofrecerles su marca a través de todo tipo de contenidos vinculados a ella, es decir, mediante estrategias de comunicación pull, en las que es el consumidor quien acude en busca de contenidos publicitarios.

Las estrategias pull se fundamentan en dos premisas: un papel más activo por parte del consumidor -que pasa de espectador a usuario- y algo que actúe como "gancho" para atraerlo, es decir, un sinfín de contenidos "en forma de artículos, informes, utilidades [...], software informático, salvapantallas, juegos, etc." (Martí, 2005:57) que permitan ofrecer al usuario ese valor añadido. 
Según Gobé, estas nuevas estrategias pueden reportar beneficios tanto para las empresas como para sus consumidores: "existe una oportunidad diferenciada de crear una relación entre las empresas y las personas que sea beneficiosa para ambos. Este modelo de sociedad 'win-win' se está convirtiendo rápidamente en el nuevo estándar en el mundo de los negocios" (Gobé, 2005).

Para las organizaciones, el beneficio principal consiste en capturar la atención de su público objetivo mediante la creación de contenidos interactivos y personalizados, siempre enfocados hacia el beneficio -entendido como facilidad, entretenimiento o comodidad- de los consumidores (Carrillo, 2005) que, así, también reciben una contraprestación a su interacción con las marcas.

Todo ello converge en la creación de productos de comunicación híbridos, que mezclan la publicidad con la información -infotainment-, con el entretenimiento -advertainment- o, como en el caso que se analizará en este artículo, con los videojuegos -advergaming-.

\section{El auge de la industria de los videojuegos}

La industria de los videojuegos ha experimentado un gran crecimiento en los últimos años. La sofisticación y la aparición de nuevas plataformas, junto con la elevada penetración de las conexiones de banda ancha en los hogares, ha ampliado y diversificado los perfiles de los videojugadores, convirtiéndolo en una alternativa de ocio de masas de gran interés para la publicidad.

El crecimiento en el consumo de videojuegos -España fue el cuarto país a nivel europeo en consumo de videojuegos (ADESE, 2008:11), con unos ingresos estimados cercanos a los 1.500 millones de euros- y la diversificación y ampliación de los perfiles de videojugadores ha hecho que los advergames empiecen a ser considerados como herramientas publicitarias por parte de empresas y organizaciones. En palabras de Martí: "Los videojuegos han pasado en apenas quince años de ser un entretenimiento de minorías (especialmente para jóvenes tecnoadictos y amantes de los ordenadores) a ser uno de los subsectores más importantes, y de mayor peso económico, en la industria del entretenimiento" (Martí, 2010:11).

Por otro lado, el segundo cambio significativo alrededor de los videojuegos ha sido su progresiva aceptación -a nivel pedagógico y social- como herramienta de aprendizaje y sociabilización -tanto por el auge de las plataformas y los videojuegos de ocio familiar como por el desarrollo de los videojuegos online multijugador-. Sin embargo, la estricta distinción entre "jugar" y "aprender" -especialmente si se trataba de videojuegos- ha sido una constante hasta hace poco tiempo:

Play and learning have been segregated from one another in contemporary schooling, further cementing their perceived disparity. Children learn while seated in desks, listening attentively to a teacher or reading from a book. This sort of valid learning is interrupted by recess, where children are allowed to play. Understood in this way, play is a distraction useful only to let off the necessary steam to allow kids (or adults) to get back to the serious business of learning (or working) (Bogost, 2008:120). 
Como señala Bogost, el hecho de que un videojuego, más allá de proporcionar entretenimiento, pueda contribuir a las estrategias de comunicación de una marca o a denunciar prácticas poco éticas por parte de empresas e instituciones -en definitiva, pueda "enseñar" algo al videojugador- representa un cambio de paradigma respecto a la visión a tradicional sobre su función, utilidad y objetivos. La naturaleza "didáctica" del soporte y su popularidad entre los más jóvenes es clave para entender el interés de los actores sociales críticos con el consumo por desarrollar anti-advergames.

\section{Hacia una definición del Advergaming}

Los advergames, al igual que otras herramientas publicitarias como el emplazamiento de producto o de marca, son formatos híbridos entre la publicidad y el entretenimiento y, por ello, "una de las ventajas de las comunicaciones de marketing a través de videojuegos es que no pueden 'eliminarse' puesto que están embebidas en el propio contenido de entretenimiento" (Martí, 2010:68).

Los advergames son, pues, una mezcla indisoluble que conjuga publicidad y videojuegos. González Díaz (2009) los define como una herramienta de comunicación enmarcada en un ambiente lúdico -el videojuego-, donde el protagonista absoluto es la marca, representada a través de su logo, su producto, sus mascotas, etc.

Este "protagonismo" del producto o marca no debe entenderse como una mera ubicación de productos o marcas en el contexto de un videojuego - una práctica conocida como publicidad in-game-, sino que se trata de que el advergame desprenda los valores del producto o marca que lo desarrolla. En palabras de Ramos (2006:45) "no se trata de insertar el producto en un videojuego ajeno, esto sería product placement, sino más la creación de un juego donde esté representado el valor de la marca o el concepto publicitario de una campaña".

Siguiendo esta misma línea, Dahl, Eagle y Báez (2006:3) y Méndiz Noguero (2010:13) argumentan que el propósito principal de los advergames consiste en ofrecer una experiencia lúdica al consumidor y conectar con él para crear una conexión emocional o un sentimiento de comunidad entre el juego y la marca en cuestión. Y es que, en tanto que los advergames son desarrollados ex profeso para difundir mensajes publicitarios (Martí, 2010:78), la trama y los escenarios del videojuego se diseñan por y para cumplir los objetivos de comunicación de una marca.

Méndiz (2010:44-45) y Clemente y Abuín (2009:1) destacan que estos videojuegos "permiten una exposición continuada del usuario ante aquello que se publicita", además de otras características como la elevada atención por parte del videojugador y su predisposición positiva, la interactividad, la memorabilidad y su potencial viralidad.

Una de las ventajas de los advergames, relacionada directamente con su memorabilidad, es el elevado tiempo de contacto que alcanza con su público objetivo, puesto que el tiempo 
medio por partida dedicado a jugar a un advergame se sitúa en los 10 minutos en un videojuego monojugador, y alcanza los 55 minutos si permite jugar en línea con otros usuarios- ("El advergaming gana adeptos", 2007).

El tiempo empleado en el videojuego, junto con la actitud activa del videojugador -que no puede perder detalle si quiere ganar la partida-, hacen que los advergames tengan un elevado índice de recuerdo (Clemente y Abuín, 2009). Y, en tanto que la marca proporciona diversión y entretenimiento de forma gratuita, la predisposición del usuario es muy positiva: "the popularity of many advergames suggests that gamers are evidently quite happy to put up with advertising in return for free entertainment-just as soap-opera fans were in the radio age" ("And now, a game from our sponsor", 2005).

Los advergames, además, transfieren la emoción del juego a la marca, creando así relaciones entre ésta y sus consumidores (Graaf Van Der y Niebor, 2003) y contribuyendo a "consolidar y difundir los universos simbólicos creados por las marcas" mediante su inmersión a través del videojuego (González Díaz, 2009, p.4).

\section{Advergames y anti-advergames como herramientas de comunicación: la simulación}

Tanto los advergames como los anti-advergame pueden utilizar dos vías para transmitir el mensaje que se pretende poner en relieve. Una de estas vías consiste en la inclusión de información en las diferentes interfaces del videojuego, cosa que no garantiza que el videojugador lea y comprenda dichos mensajes. La segunda vía, más propia del género de los videojuegos, se basa en utilizar las simulaciones, mediante las cuales se recrean determinados eventos o situaciones para hacer que el videojugador aprenda mientras actúa (Lavigne, 2007), de forma que se garantiza su comprensión sobre las consecuencias de sus acciones. En la opinión de Bogost, se puede aprender no solo a través del contenido del propio videojuego, sino con el propio acto de jugar: "games teach through their procedural rhetoric: players develop strategies for learning and problem-solving by 'reading' the game's rhetoric" (entrevistado en Jansz, 2008:796).

Los videojuegos, como define Bogost, "son una forma de comunicación diferente a la de los otros medios: no sólo transmiten mensajes, sino que simulan experiencias" (entrevistado en García, 2008). Los advergames y los anti-advergames, por lo tanto, facilitan la asimilación de mensajes, actitudes y conductas, ya que ayudan a aprender de forma activa y amena, sin riesgos, escogiendo libremente las alternativas y mediante la identificación con los personajes.

La simulación permite que el videojugador experimente roles e interrelacione las acciones con sus consecuencias, generando empatía y contextualizando los acontecimientos a nivel espacio-temporal de manera muy superior a lo que puede lograr, por ejemplo, leer un artículo en la prensa (Ochalla, 2007). 
Mientras que en el caso de los advergames la simulación puede servir para demostrar el funcionamiento de un determinado producto -como es el caso de los advergames desarrollados por compañías automovilísticas en los que se puede "conducir" un coche virtualmente-, los anti-advergames suelen plantear al videojugador el reto de cómo actuar ante un escenario "problemático" -el que se pretende denunciar o criticar- (Jared:2008).

\section{De la contracultura a los anti-advergames}

El capitalismo y el consumismo han sido dos de los principales blancos de la crítica antisistema. No es extraño ni novedoso, pues, que la publicidad y el marketing -expresiones por antonomasia de la economía de libre mercado- hayan centrado la atención de todo tipo de movimientos contraculturales (Rom y Sabaté, 2006:140), manifestando su disconformidad mediante parodias y críticas de piezas publicitarias -en un principio mediante los anuncios gráficos, y más recientemente también en piezas audiovisuales-.

John Fiske, en el libro Television Culture (1987), utilizó el concepto de "democracia semiótica" para designar las formas en que las audiencias crean sus propias interpretaciones de los contenidos de entretenimiento de masas (Walker, 2006). A partir de este concepto, Katyal profesora de derecho en la Universidad de Fordham- acuñó el término "desobediencia semiótica", que define como "a number of different approaches to visual, actual, and verbal representation, including vandalizing, subverting, and "recoding" certain kinds of intellectual, real, government, and private property for public use and expression" (Katyal, 2006:493).

Este tipo de prácticas de carácter subversivo hacia la publicidad, también llamadas antiadvertising, adbusting o culture jamming (Jared, 2008) cuentan con décadas de trayectoria. En Canadá incluso cuentan con la Adbuster Media Foundation, una fundación contra el consumismo, que edita bimensualmente la revista Adbusters (www.adbusters.org). Dicha publicación, con una tirada de 120.000 ejemplares, gira alrededor de dos ejes temáticos principales: la colonización de espacios por parte del marketing y las tecnologías de la información y la comunicación, y la degradación medioambiental derivada del consumismo (Rumbo, 2002:138).

Como puede observarse, estos conceptos coinciden con los objetivos de los anti-advergames y, por ello, pueden considerarse sus antecedentes más directos. Como explica Radtke: "Whereas the anti-advergame is a recent trend, other mediums have previously tried similar approaches. The most notable is the subvertising movement, which twists a brand's well known advertisement campaign into a negative critic, hoping to lead to a consumer's perspective change" (Radtke, 2008:87).

La aparición de los anti-advergames, en definitiva, muestra como la sofisticación, los objetivos y las tácticas de los publicitarios y los "anti-publicitarios" han evolucionado en paralelo (Walker, 2006). El supuesto peligro social que para algunos alarmistas podría representar lo contracultural, queda desdramatizado y devaluado desde el mismo momento 
en que se puede decir que la contracultura es, además de un alimento básico del consumo, una foma de generar ideas para el marketing (Sabaté y Rom, 2008: 70).

\subsection{Anti-advergames: definición y características}

Si los advergames son videojuegos encaminados a promocionar productos o marcas comerciales, los anti-advergames pretenden, fundamentalmente, promover determinadas ideas y actitudes. Como sugiere su nombre, sus objetivos se oponen frontalmente a los fines de los advergames y, por ello, lo que se busca es criticar un producto, una marca o determinados procedimientos de negocio de una corporación. Sin embargo, existe otra modalidad de anti-advergame que se aleja de esta vertiente anti-comercial para centrarse en la crítica de sucesos de actualidad o de ideas políticas, económicas y sociales -y cuya sátira, por lo tanto, se dirige a instituciones, gobiernos o incluso hacia elementos de la propia cultura-. Como explica Radtke:

The more well known is the anti-advergame, which subverts the advergame mechanics to demote a brand for its wrong doings. The second stream focuses in more general social, economics or ecological aspects. Although the first stream involves a direct campaign against a brand, the term anti-advergame is often used to describe both streams (Radtke, 2008:85).

Como es habitual en los formatos y herramientas de reciente aparición, no existe consenso sobre la denominación más adecuada para estas herramientas. Así, encontramos que para Terdiman (2006) y Jared (2008), cuyas definiciones destacan el componente "anti-compañía" de estos videojuegos, el término elegido es anti-advergames. Por su parte, Ian Bogost, quien prioriza el componente persuasivo-argumentativo de estos juegos, considera que el término persuasive games es el idóneo para etiquetar todo tipo de videojuegos que pretendan influenciar al videojugador, independientemente de si se trata de videojuegos educativos, de temática grave, o bien críticos con empresas e instituciones (Bogost, 2007:57, en Jansz, 2008:795).

Otro de los términos empleados, newsgaming, fue acuñado por un grupo de artistas y desarrolladores de videojuegos asociados en un sitio web con el mismo nombre (www.newsgaming.com), que sirve de plataforma para distribuir videojuegos sobre cuestiones de actualidad. Tal y como explican en su sitio web, los videojuegos pueden, además de entretener, fomentar el pensamiento crítico: "traditionally, videogames have focused on fantasy rather than reality, but we believe that they can be a great tool for better understanding our world"

Terdiman (2006) define los anti-advergames como sátiras hacia grandes empresas que cuestionan sus políticas corporativas en diferentes ámbitos, desde los bajos salarios de sus empleados hasta sus políticas medioambientales. Jared, cuya definición sigue esta misma línea, añade que suele tratarse de videojuegos gratuitos que pueden jugarse a través del sitio web de la marca que los ha impulsado (Jared, 2008). 
Para Paolo Pedercini, fundador de La Molleindutria, los anti-advergames son una reapropiación de los videojuegos que combina el activismo mediático con la crítica a los videojuegos. Según este autor, los videojuegos son herramientas muy útiles para explicar y representar sistemas complejos -a nivel económico, político o social- en los que intervienen diversos actores que interactúan entre sí. Por ello, considera que el objetivo de los antiadvergames consiste en "spread unpopular ideas using video games, taking advantage of the viral infusion of content on the net and at the same time investigating the relationship between ideology and entertainment" (Alexander, 2010).

Ian Bogost -socio fundador de la compañía Persuasive Games y profesor en el Georgia Institute of Technology- considera que, mientras que las campañas publicitarias de anunciantes, gobiernos y organizaciones sirven para mostrar lo que quieren que vean sus audiencias, el cometido de los anti-advergames es precisamente mostrar aquello que pretenden esconder. Y los videojuegos, según su punto de vista, son una herramienta particularmente eficaz para ello: "videogames are particularly good at exposing the underlying logics of these organizations -how they work and what's wrong with it" (entrevistado en Terdiman, 2006).

Su aparición, según Bogost, responde a las demandas de una parte de videojugadores interesados en videojuegos que, además de brindarles entretenimiento, les ofrecieran una cierta profundidad en su significado: "we wanted to show them you can produce a game that is just as engaging as anything out there, but also has a positive message and influence" (entrevistado en Ochalla, 2007:4). En la misma línea se manifiesta Seggerman, quien considera que se trata de una evolución natural del género, como ocurrió con la televisión: "'It took a while for film to start taking a look at serious issues [...]. We didn't see documentaries come to the fore until the late 60 s or early 70 s. So it's going to be a little while before serious games hit their stride and gain mainstream attention" (entrevistado en Ochalla, 2007:4).

Sin embargo, otros autores consideran que el carácter lúdico o de entretenimiento es un requisito fundamental para que un anti-advergame funcione. En palabras de Radtke (2008:87): "besides passing a message, anti-advergames have to be fun and challenging games. Otherwise, they will fail on passing the massage, as the basic requirement is that people have to actually play it to get a message". Además, un videojuego divertido o entretenido es capaz de centrar la atención de los videojugadores en lo que sucede en la pantalla, por lo que es más probable que el videojugador se interese por el mensaje que recibe (Boyd, 2006).

En definitiva, se puede considerar que los anti-advergames -también llamados persuasive games, newsgames o serious games- son videojuegos creados para que el videojugador experimente, mediante su interacción con una realidad simulada presentada de forma (pseudo)lúdica, un mensaje que pretende censurar, desacreditar o menospreciar marcas, 
productos, ideas o comportamientos -tanto de empresas como de organizaciones o instituciones- que se consideran reprochables.

\subsection{Advergames vs. Anti-advergames}

Una de las principales diferencias entre los advergames y los antiadvergames es su presupuesto. Gonzalo Frasca, desarrollador de ambos tipos de videojuegos, considera que los elevados costes que supone desarrollar un videojuego es uno de los principales escollos que deben salvar los anti-advergames: "Political games generally do not have a financial return, and that makes it particularly hard to produce them with the same quality as commercial work" (entrevistado en Ochalla, 2007). La escasez de presupuesto también puede entorpecer la distribución y la difusión de estos videojuegos, dejándolos a merced de la publicity y/o la viralidad que sean capaces de generar. En palabras de Radtke (2008:87):

Ultimately, the final aspect that usually undermines those games efforts is their limited distribution and, ironically, lack of advertisement, so they can reach a wider user range. Besides gaming industry insiders and researchers, games trying to deliver a message are unknown to the mainstream market that is not actively related to the message those games try to deliver. Making these games fun is a mandatory step, but making them reach the masses is just as important.

Swain, profesor en la USC School of Cinematic Arts, sugiere que el problema presupuestario se debe, mayoritariamente, a la reciente aparición de este género: "the field of political/activist games is very young. We need some success stories to prove our value because right now political games mostly grab headlines and have little real impact" (entrevistado en Ochalla, 2007).

Para otros autores, el propósito de los anti-advergames no depende tanto de su desarrollo artístico y técnico sino una buena idea que logre transmitir su mensaje. Prueba de ello, como argumenta García (2008), es que pese a las innumerables mejoras técnicas que ha experimentado el sector de la animación y los videojuegos, títulos clásicos como Super Mario Bros., Tetris o Pacman siguen considerándose entre los mejores videojuegos de la historia:

Frente a un mercado que se desarrolla más rápido que cualquier otra industria cultural, ganaron programas de hace dos décadas, prueba de que todavía no hay sofisticación que valga más que una buena idea. $Y$ no es casualidad que, bordeando tanta superproducción, esté floreciendo un movimiento de programadores independientes que están seguros de que lo más sorprendente aún está por venir (García, 2008).

Como se analizará a continuación, mientras que algunos anti-advergames, como McVideoGame, realizan una crítica global a las políticas de gestión de la empresa, otros centran la atención del videojugador en un único mensaje -como es el caso de September 12-. En este sentido, Jared (2008:3) sostiene que los anti-advergames, más que un ataque ambiguo al modo de proceder de una empresa, deben centrarse en ofrecer un mensaje claro. 


\section{El caso de McDonald's Video Game y September 12}

\subsection{McDonald's Video Game: ¿Qué hay detrás de una hamburguesa?}

El primero de los casos que se analizarán corresponde a un anti-advergame desarrollado en el año 2006 por La Molleindustria (www.molleindustria.org), un equipo de artistas, diseñadores y programadores italianos que se dedican a diseñar videojuegos con implicaciones políticas y sociales.

El videojuego, llamado McDonald's Video Game, es accesible desde la dirección www.mcvideogame.com y está traducido a nueve idiomas. En un primer momento, el videojugador no halla ninguna evidencia de crítica a esta compañía sino que, como describe Radtke (2008:86), "the game's website is designed to lure the players as if it was an actual McDonald's website, an illusion destroyed later by the satirical and often acid comments on the corporation before the player starts the game".

Antes de empezar la partida, sin embargo, un mensaje advierte al videojugador de que se trata de "una parodia digital de McDonald's" cuyo propósito es "el entretenimiento y la educación", al tiempo que declinan cualquier responsabilidad legal al alegar que ninguno de los creadores está afiliado a la compañía y que "el contenido de este juego no debe ser tomado de ninguna forma como verdadero".

El objetivo del videojuego es dirigir la compañía McDonald's, controlando todos y cada una de los procesos que intervienen para la elaboración de las conocidas hamburguesas. Para ello, la interfaz del juego se divide en cuatro secciones: el sector agrícola, la granja de engorde, el establecimiento de comida rápida y la sede central. En cada escenario se deben llevar a cabo prácticas poco éticas si se quiere evitar que la empresa se declare en bancarrota y, en consecuencia, perder la partida. Entre estas prácticas se incluye ampliar los cultivos de soja deforestando la selva, acelerar el crecimiento de las reses mediante hormonas, presionar a los empleados de las tiendas para mejorar su productividad o sobornar a climatólogos, políticos y nutricionistas desde la oficina de Relaciones Públicas, entre muchas otras. En definitiva, y tal como se explica en la pantalla inicial, se trata de que el videojugador descubra "todos los sucios secretos que nos hacen ser una de las compañías más grandes del mundo"

Los videojugadores deben enfrentarse a continuas tomas de decisiones, tanto a nivel empresarial como en el plano de la moralidad (Bogost, 2008:126). En el tutorial del videojuego, por ejemplo, se explica que la selva tropical "puede talarse para dejar sitio a pastos y cultivos". Diversos públicos de interés -ecologistas, anti-sistema, etc.- pueden censurar las decisiones tomadas durante el transcurso del videojuego, por lo que el jugador debe también gestionar campañas de relaciones públicas o ejercer presión desde sus lobbies para neutralizar estas críticas. 
Este anti-advergame, por lo tanto, pretende subrayar de forma entretenida e irónica que los pilares que sustentan al gigante de las hamburguesas son la codicia y la corrupción. En palabras de Bogost: "The McDonald's Videogame mounts a procedural rhetoric about the necessity of corruption in the global fast food business, and the overwhelming temptation of greed, which leads to more corruption" (2008:127).

Sin embargo, cabe valorar si el hecho de que la multinacional McDonald's haya sido acusada en repetidas ocasiones -tanto en los medios de comunicación, especialmente mediante el documental SuperSize Me (2004), como en los tribunales- puede llegar a disminuir la eficacia de este anti-advergame, puesto que la crítica a esta corporación es ya casi un lugar común y ello reduce el impacto que puede causar en los videojugadores (Jared, 2008).

\subsection{September 12: misiles para reflexionar.}

September 12 es un anti-advergame lanzado en 2003 por el investigador y desarrollador de videojuegos Gonzalo Frasca. Su objetivo es que el videojugador reflexione sobre cuestiones sociales complejas. En este caso, pues, la crítica no se dirige hacia una empresa sino hacia las consecuencias de determinadas acciones políticas (Terdiman, 2006), en concreto la guerra contra el terrorismo iniciada por Bush a raíz de los atentados del 11-S.

Antes de empezar la partida, un mensaje en la pantalla presenta el videojuego -en el que coexisten dos tipos de personajes: terroristas y civiles- y explica escuetamente su única regla: se puede optar entre hacer estallar misiles o no hacerlo. La partida no tiene fin, y tampoco se puede ganar ni perder porque, tal y como explican, su objetivo es presentar una simulación que ayude a "explorar algunos aspectos de la guerra contra el terrorismo".

La interfaz del videojuego consta de un único escenario que simula un pueblo anónimo de Oriente Medio repleto de personas -civiles y terroristas- que recorren sus calles. El videojugador controla una mirilla para dirigir sus proyectiles, supuestamente con el objetivo de eliminar únicamente a los terroristas, pero el tiempo que transcurre entre el lanzamiento y la explosión, junto con el movimiento constante de los personajes, hace que irremediablemente mueran numerosos civiles y se destruyan edificaciones del pueblo.

Sin embargo, las consecuencias de estos "daños colaterales" van mucho más allá, puesto que los familiares de los civiles muertos, después de guardar luto por sus pérdidas, se convierten en terroristas. En palabras de Terdiman (2006): "The fun quickly turns political, however, as villagers mourning friends and relatives accidentally killed by the missiles morph into terrorists themselves".

Jansz (2008:795) describe el desafortunado círculo vicioso que propicia el juego: "They can succeed by firing missiles, but this inevitably causes collateral damage in the Middle-Eastern village. As a result, ordinary citizens in the game turn into terrorists and players need to increase the frequency of their fire to kill their ever-increasing numbers". Es decir, cuantos 
más misiles se lanzan para eliminar terroristas, más civiles mueren; cuantos más civiles mueren, más familiares se convierten en terroristas $y$, cuantos más terroristas, más ofensivas militares serán "necesarias" para cumplir el supuesto objetivo inicial de vencer al terrorismo.

Las propias reglas del juego, pues, conducen a una moraleja clara: "Violence begets more violence, and the nonprecision weapons of American "precision warfare" bear significant consequence in the form of innocent lives lost" (Bogost, 2006:168).

Así pues, por sus particulares características - la imposibilidad de ganar/perder una partida que no termina nunca- y por su temática candente, este anti-advergame consigue impactar al videojugador con un mensaje contundente y autocrítico.

\section{Conclusiones}

La paradoja de los contrarios es que no son necesariamente excluyentes. Dos espejos enfrentados se limitan a proyectar la misma imagen invirtiendo el orden de izquierda y derecha. Es más, aunque la finalidad de los anti-advergames sea denunciar determinados comportamientos o acciones por parte de empresas e instituciones, algunos expertos en marketing, como Brad Scott, consideran que posiblemente estas compañías -a las que el videojuego pretende denunciar- puedan llegar incluso a beneficiarse de la notoriedad que les brinda un determinado anti-advergame (Terdiman, 2006). Lo cierto es que, como argumenta Binay (2005:96) en su tesis doctoral, no existen evidencias de que las campañas antipublicidad ayuden a prevenir el consumismo o cambien las actitudes de los individuos respecto a una marca, a no ser que coincidan con una motivación real de su público objetivo y emitan un mensaje claro. En caso contrario, incluso pueden producir un efecto parecido a las campañas publicitarias originales de la marca en cuestión.

En la opinión del experto en videojuegos Matteo Bittanti, los buenos anti-advergames funcionan del mismo modo que las buenas películas: no sólo entretienen sino que consiguen que conectes con su mensaje (entrevistado en Boyd, 2006).

No pretendemos afirmar que los advergames son la nueva piedra filosofal de la publicidad. Aunque es cierto que este tipo de videojuegos pueden ser una forma amena y entretenida de argumentar y fomentar la reflexión, aún no se han realizado estudios que demuestren y/o valoren sus posibles efectos sobre las marcas o ideas hacia las que enfocan sus críticas. Sin embargo, los casos de anti-advergames analizados revelan algunas cuestiones de gran interés para su aplicación comercial.

En primer lugar, los anti-advergames, con un presupuesto muy limitado, se concentran en la transmisión de una mensaje crítico, contundente y provocador. Se centran en el valor del contenido comunicativo, mientras que los advergames, en ocasiones, priorizan el componente estético y lúdico del juego para ofrecer una experiencia casi exclusivamente al 
entretenimiento, olvidando que cualquier estrategia publicitaria debe centrarse, en primer lugar, en definir el mensaje que se pretende transmitir a un determinado público objetivo.

En segundo lugar, los anti-advergames analizados demuestran la utilidad de la simulación como herramienta de aprendizaje para abordar argumentos socialmente complejos, alejados de la percepción de trivialidad tradicionalmente asociada a los videojuegos. De ello se infiere que sería posible extrapolar estos mecanismos no sólo a productos de entretenimiento contraculturales, sino también al servicio de organizaciones y administraciones, especialmente en campañas de sensibilización en temas de salud, prevención de adicciones, etc. La filosofía de los anti-advergames analizados pone especial interés en la identificación con una situación o un personaje y en la libre elección de alternativas: en ninguno de los dos casos se puede ganar la partida, sino únicamente evidenciar las consecuencias derivadas de la toma de decisiones. Estos elementos propios de los anti-advergames también podrían extrapolarse a advergames de carácter más pedagógico. En definitiva, nos encontramos ante una herramienta con un gran potencial que merece ser estudiada a fondo para evaluar con rigor su alcance y su eficacia. 


\section{REFERENCIAS BIBLIOGRÁFICAS}

ADESE - SOCiación Española de Distribuidores y Editores de Software (2008): "Anuario ADESE 2008", en http://www.adese.es/pdf/anuario-memoria-2008.pdf (consultado: 22/6/2010).

AleXANDER, Leigh (2010): "GDC: Molleindustria's Pedercini on 'Reappropriating' Video Games", en Gamasutra: the Art and Business of Making Games (10/3/2010), en

http://www.gamasutra.com/view/news/27623/GDC Mollendustrias Pedercini_On_ Reappropriating Video Games.php (consultado: 2/5/2010).

"AND NOW, A GAME FROM OUR SPONSOR", en The Economist (9/6/2005), en http://www.economist.com (consultado: 9/3/2010).

BINAY, Ayse (2005): "Investigating The Anti-Consumerism Movement in North America: The Case of Adbusters" (tesis doctoral), en: http://www.lib.utexas.edu/etd/d/2005/binaya18966/binaya18966.pdf (consultado: 2/7/2010).

Bogost, Ian (2008): "The Rhetoric of Video Games", en SALEN, Katie (ed.): The Ecology of Games: Connecting Youth, Games, and Learning. The John D. and Catherine T. MacArthur Foundation Series on Digital Media and Learning. Cambridge, MA, The MIT Press, pp. 117-140.

Bogost, Ian (2007): Persuasive Games: The Expressive Power of Videogames. Cambridge, MA, MIT Press.

Bosco, Roberta; CaldanA, Stefano (2006): "Activismo y juego, en una parodia inspirada en la multinacional McDonald's", en El País (20/4/2006), en: http://www.elpais.com/articulo/portada/Activismo/juego/parodia/inspirada/multina cional/McDonald/s/elpcibpor/20060420elpcibpor_2/Tes (consultado: 2/5/2010).

Boyd, Clark (2006): "Hackers re-invent political protests", en BBC News (6/10/2006), en: http://news.bbc.co.uk/2/hi/technology/3707572.stm (consultado: 12/5/2010).

CARRILlo, María Victoria (2005): "El consumidor: claves del comportamiento y nuevo entorno interactivo". En: Victoria, Juan Salvador (coord.) (2005): Reestructuras del sistema publicitario. Barcelona, Ariel, pp. 221-238.

Clemente, Jorge; Abuín, Natalia (2009): "La nueva publicidad en videojuegos y dispositivos móviles: advergaming 2.0", en Actas del I Congreso Internacional Brand Trends. Valencia, Universidad Cardenal Herrera CEU.

"El ADVERgaming GANA ADEPTOS" (2007), en Marketingnews.com (27/3/2007), en http://www.marketingdirecto.com/actualidad/digital/el-advergaming-gana-adeptos (Consultado: 12/3/2010). 
GARCíA, Facundo (2008): "Llegó la hora de abrir el juego", en Página 12, (10/8/2008), en: http://www.pagina12.com.ar/diario/suplementos/espectaculos/210876-2008-08-10.html (consultado: 3/6/2010).

GoBÉ, Marc (2005): Branding emocional. Barcelona, Divino Egg Publicaciones.

GonzÁlEZ DÍAZ, Cristina (2009): "Advergaming aplicado a las estrategias comunicativas dirigidas al target infantil", en Actas del I Congreso Internacional Brand Trends. Valencia, Universidad Cardenal Herrera CEU.

VAN DER GraAF, Shenja; Nieborg, David B. (2003). "Together we brand: America's Army". En: CoPIER, Marinka; RAESENS, Joost (eds.) (2003): Level Up: Digital Games Research Conference. Utrecht, Holland, Universiteit Utrecht, pp. 324-338.

JANSZ, Jeroen (2008): "The paratextual pleasures of reading about playing video games", en New Media \& Society, vol. 10, no 5 (October 2008), p.793-801.

JARED, Newman (2008): "Persuasion is Futile: The Trouble with Anti-Advergames", en The Escapist Magazine, núm. 137, en: http://www.escapistmagazine.com/articles/view/issues/issue_137/2938-

Persuasion-is-Futile (consultado: 11/5/2010).

KATYAL, Sonia (2006): "Semiotic disobedience", en Washington University Law Review, vol. 84, no 2, pp.489-571.

Lavigne, Chris (2007): "Super Chick Sisters and the Rise of the Message Game", en The Escapist, no 128, en:

http://www.escapistmagazine.com/articles/view/issues/issue 128/2725-Super-

Chick-Sisters (consultado: 10/6/2010).

MARTí, José (2010): Marketing y videojuegos: product placement, in-game advertising y advergaming. Madrid, ESIC.

MARTÍ, José (2005): Publicidad y entretenimiento en la web. Madrid, Ra-Ma.

MÉndiz Noguero, Alfonso (2010): "Advergaming. Concepto, tipología, estrategias y evolución histórica", en Revista Icono 14, no 15 (enero 2010), pp. 37-58, en: www.icono14.net (consultado: 5/4/2010).

Ochalla, Bryan (2007): "Who Says Video Games Have to be Fun? The Rise of Serious Games", en Gamasutra: The Art \& Business of Making Games (29/6/2007), en:

http://www.gamasutra.com/view/feature/1465/who says video games have to b e .php?page $=1$ (consultado: 14/6/2010).

RADTKE, Paulo V. W. (2008): "Games with an Agenda: Entertainment for Consciousness Building", en VIII Symposium on Computer Games and Digital Entertainment Proceedings. Belo Horizonte (Brasil), Sociedade Brasileira de Computação. 
RAmOS, Marina (2006): "Cuando la marca ofrece entretenimiento: aproximación al concepto de advertainment", en Questiones publicitarias: revista internacional de comunicación y publicidad, vol 1, no 11 (2006), pp. 33-49.

Rom, Josep A.; SABATÉ, Joan (2006): "Publicitat i contracultura", en Tripodos, no 18, pp.139-156.

RumBo, Joseph E. (2002): "Consumer Resistance in a World of Advertising Clutter: the case of Adbusters", en Psychology and Marketing, vol. 19, n० 2 (febrero 2002), pp.127-148.

SABATÉ, Joan; Rom, Josep A. (2008): "Marketing, publicidad y consumo contracultural". En: ReY, Juan (ed.) (2008): Publicidad y sociedad. Un viaje de ida y vuelta. Sevilla, Comunicación Social.

Terdiman, Daniel (2006): "Games that stick it to 'The Man"', en CNET News (3/2/2006), en http://news.cnet.com/Games-that-stick-it-to-The-Man/21001043_3-6035028.html?tag=mncol (consultado: 1/6/2010).

WALKer, Rob (2006): "Gaming the System", en The New York Times Magazine $(3 / 9 / 2006)$,

en:

http://www.nytimes.com/2006/09/03/magazine/03wwln consumed.html?ex=1314

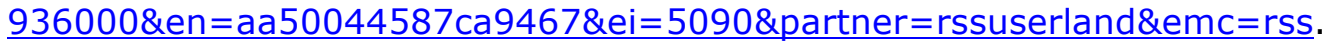

(consultado: 23/5/2010).

[Recibido: 22 de septiembre de 2010. Aceptado (con indicación de cambios): 25 de mayo de 2011. Recepción del artículo corregido: 6 de junio de 2011.] 